\title{
Mützchen blau
}

\author{
Zwar steht die digitale Barrierefreiheit im Gesetz. \\ Doch mit der Umsetzung hapert es. \\ Michaela Endemann
}

„Am ersten Blue Beanie Day im November 2007 und seither jedes Jahr am 30. November tragen Mann und Frau in Amerika und weltweit blaue Mützchen, die ,blue beanies', um ihre Unterstützung für Webstandards und Accessibility zu demonstrieren“, sagt Robert Lender in seinem Blog. Mit seiner blauen Strickmütze setzt er sich in Österreich Jahr für Jahr als Avatar in den sozialen Medien für dieses Thema ein.

Digitale Barrierefreiheit ist auch heute keine Selbstverständlichkeit. Susanne Buchner-Sabathy ist Expertin für barrierefreie Kommunikation und Vorsitzender-Stellvertreterin des Vereins Accessible Media: „Die Diskussionen rund um die bauliche Barrierefreiheit und um das Ende der diesbezüglichen Übergangsfrist im Jahr 2016 ließen leider oft vergessen, dass Webseiten in Österreich bereits seit 2006 barrierefrei sein müssen. Damit gilt in Österreich seit 2006 sowohl für öffentliche Stellen als auch für privatwirtschaftliche Anbieter elektronischer Informationen die Verpflichtung zur digitalen Barrierefreiheit.“

\section{Österreichischer Haken}

2006 hat Österreich die UN-Behindertenkonvention unterzeichnet, 2019 die entsprechende EU-Richtlinie durch das Web-Zugänglichkeitsgesetz (WZG) umgesetzt, wodurch auch das Internet insbesondere für Menschen mit Behinderungen besser zugänglich gemacht werden soll. Allerdings mit einem österreichischen Haken, so Klaus Höckner, Stellvertretender Vorstandsvorsitzender der Hilfsgemeinschaft der Blinden und Sehschwachen Österreichs: „Wir haben wieder einmal ein Problem mit dem Föderalismus, d.h. es wurden neun länderspezifische und eine bundesweite Umsetzung durchgeführt." Da das Gesetz keine amtswegige Überprüfung vorsieht, wurde, wie es die EU-Richtlinie vorsieht, eine nationale Monitoringstelle installiert. „So kann man Menschen, die sich diskriminiert fühlen, nur raten, die Möglichkeit eines Schlichtungsverfahrens anzustreben", sagt Buchner-Sabathy.

\section{Design vor Funktion}

Barrierefreiheit stehe allerdings immer noch nicht im Fokus und Klaus Höckner mahnt hier auch die Betreiber öffentlicher Websites: „Design geht immer noch vor Funktionalität und die Einbeziehung der Zielgruppe ist leider immer noch ein Fremdwort." Das Webzugänglichkeitsgesetz und der European Accessibility Act ließen jedoch die Hoffnung aufkeimen, dass sich die Situation in den nächsten Jahren

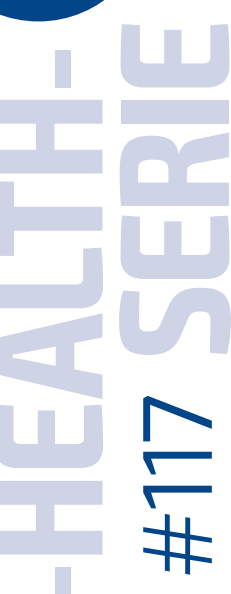

Die vier Säulen einer barrierefreien Website:

- Wahrnehmbarkeit - es muss erkennbar sein, was sich darauf befindet

Bedienbarkeit - man muss navigieren können;

- Verständlichkeit - man muss erkennen können, was das Ziel und der Sinn ist;

- Robustheit - die Technik muss modern sein und auf allen Endgeräten funktionieren.

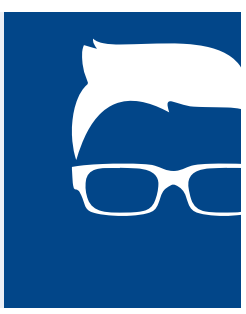

verbessern werde. Buchner-Sabathy empfiehlt in diesem Zusammenhang, „Barrierefreiheit als ein wichtiges Ziel bei der Entwicklung digitaler Angebote zu definieren und das Thema früh im Entwicklungsprozess zu verankern“. Das bedeute, die AccessibilityKompetenzen von Entwicklern und Entwicklerinnen zu stärken und digitale Angebote bereits im Entwicklungsprozess von Menschen mit unterschiedlichen Behinderungen testen zu lassen. Das spare Ärger und Aufwand und Kosten im Nachhinein. „Ich bin überzeugt, dass es fast immer mangelndes Bewusstsein ist, das zu unzugänglichen digitalen Angeboten führt, nicht Ignoranz oder die Absicht, Menschen auszuschließen. Darum ist es so wichtig, über dieses Thema zu informieren." Blaue Mützen wird es also auch in Zukunft noch weiterhin geben müssen.

\section{DR. MICHAELA ENDEMANN}

endemann@gesundheitswirtschaft.at 